\title{
Muscle Krüppel-like factor 15 regulates lipid flux and systemic metabolic homeostasis
}

\author{
Liyan Fan, ${ }^{1,2}$ David R. Sweet, ${ }^{1,2}$ Domenick A. Prosdocimo, ${ }^{1,3}$ Vinesh Vinayachandran, ${ }^{1}$ Ernest R. Chan, ${ }^{4}$ Rongli Zhang, ${ }^{1}$ \\ Olga Ilkayeva, ${ }^{5}$ Yuan Lu, ${ }^{1,6}$ Komal S. Keerthy, ${ }^{1}$ Chloe E. Booth, ${ }^{1}$ Christopher B. Newgard, ${ }^{5,7}$ and Mukesh K. Jain ${ }^{1}$
}

'Case Cardiovascular Research Institute, Case Western Reserve University, and Harrington Heart and Vascular Institute, University Hospitals Cleveland Medical Center, Cleveland, Ohio, USA. ${ }^{2} \mathrm{Department} \mathrm{of}$ Pathology, Case Western Reserve University, Cleveland, Ohio, USA. ${ }^{3}$ The Webb Law Firm, Pittsburgh, Pennsylvania, USA. ${ }^{4}$ Institute for Computational Biology, Case Western Reserve University, Cleveland, Ohio, USA. ${ }^{5}$ Sarah W. Stedman Nutrition and Metabolism Center and Duke Molecular Physiology Institute, Duke University School of Medicine, Durham, North Carolina, USA. ${ }^{6}$ Charles River Laboratories, Ashland, Ohio, USA. 'Departments of Medicine and Pharmacology and Cancer Biology, Duke University School of Medicine, Durham, North Carolina, USA.

\begin{abstract}
Skeletal muscle is a major determinant of systemic metabolic homeostasis that plays a critical role in glucose metabolism and insulin sensitivity. By contrast, despite being a major user of fatty acids, and evidence that muscular disorders can lead to abnormal lipid deposition (e.g., nonalcoholic fatty liver disease in myopathies), our understanding of skeletal muscle regulation of systemic lipid homeostasis is not well understood. Here we show that skeletal muscle Krüppel-like factor 15 (KLF15) coordinates pathways central to systemic lipid homeostasis under basal conditions and in response to nutrient overload. Mice with skeletal muscle-specific KLF15 deletion demonstrated (a) reduced expression of key targets involved in lipid uptake, mitochondrial transport, and utilization, (b) elevated circulating lipids, (c) insulin resistance/glucose intolerance, and (d) increased lipid deposition in white adipose tissue and liver. Strikingly, a diet rich in short-chain fatty acids bypassed these defects in lipid flux and ameliorated aspects of metabolic dysregulation. Together, these findings establish skeletal muscle control of lipid flux as critical to systemic lipid homeostasis and metabolic health.
\end{abstract}

\section{Introduction}

Beyond the structural and locomotive functions classically ascribed to skeletal muscle, this tissue represents the largest metabolic organ by mass and is a major site for the metabolism of lipids, carbohydrates, and amino acids (1). In response to various physiologic demands, skeletal muscle quickly modulates these metabolic processes to meet its energetic needs and simultaneously influence systemic nutrient homeostasis. As such, skeletal muscle dysfunction critically contributes to metabolic disease, most familiarly through disturbing glucose homeostasis (2). Interestingly, dysfunction of skeletal muscle can also impact lipid homeostasis. For example, individuals suffering from skeletal muscle pathologies such as Duchenne muscular dystrophy and sarcopenia have greatly increased risk of nonalcoholic fatty liver disease (NAFLD), and increases in skeletal muscle mass can either slow down or resolve overstorage of liver fat (2-5). However, the importance of skeletal muscle in systemic lipid balance and metabolic disorders and the molecular pathways controlling such functions remain incompletely understood.

The zinc-finger transcription factor Krüppel-like factor 15 (KLF15) has been identified as an important regulator and effector of both intra- and interorgan metabolic processes. KLF15 is highly expressed in metabolically active tissues (e.g., liver, heart, and

Conflict of interest: The authors have declared that no conflict of interest exists. Copyright: @ 2021, American Society for Clinical Investigation.

Submitted: April 23, 2020; Accepted: December 1, 2020; Published: February 1, 2021.

Reference information: J Clin Invest. 2021;131(4):e139496.

https://doi.org/10.1172/JCl139496. skeletal muscle), is involved in the metabolism of all major macronutrients across diverse tissues, and exhibits circadian rhythmicity that synchronizes internal molecular events with environmental cues (6-9). Furthermore, genome-wide studies have identified human KLF15 variants with increased body mass index and diabetic cardiomyopathy, suggesting a role for this transcription factor in the development of metabolic disease $(10,11)$.

In this study, we generated a skeletal muscle-specific KLF15 deletion (K15-SKO) mouse model to investigate the role of this key metabolic regulator in systemic metabolic homeostasis. We demonstrate that KLF15 critically orchestrates lipid metabolism networks in skeletal muscle and has a profound impact on distant organs, contributing to adiposity and NAFLD development. These studies highlight an under-appreciated role for skeletal muscle as a critical modulator of systemic lipid metabolism and highlight the importance of muscle-liver crosstalk in metabolic disease. Finally, we show that a diet rich in short-chain fatty acids (SCFAs) can bypass defects in skeletal muscle lipid handling and improve metabolic health in the context of aberrant lipid handling.

\section{Results and Discussion}

Klf15 is highly expressed in skeletal muscle relative to other metabolically active organs, although differences between various skeletal muscle beds vary minimally regardless of fiber type composition (Supplemental Figure 1, A and B; supplemental material available online with this article; https://doi.org/10.1172/ JCI139496DS1). To study the skeletal muscle intrinsic role of KLF15 in regulating systemic metabolism, we generated a K15SKO mouse by mating the KLF15 ${ }^{\mathrm{f} / \mathrm{l}} \mathrm{l}$ line with the myogenin-Cre 
A

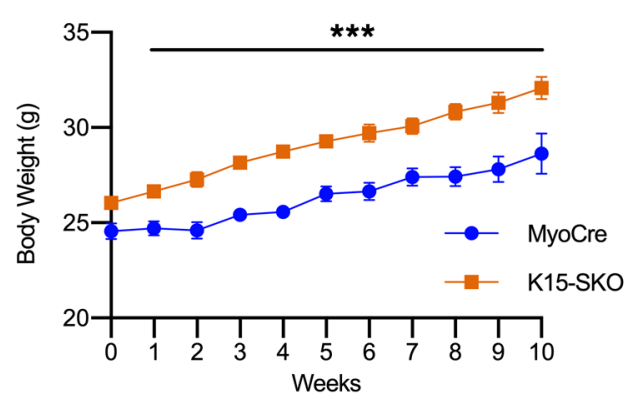

B

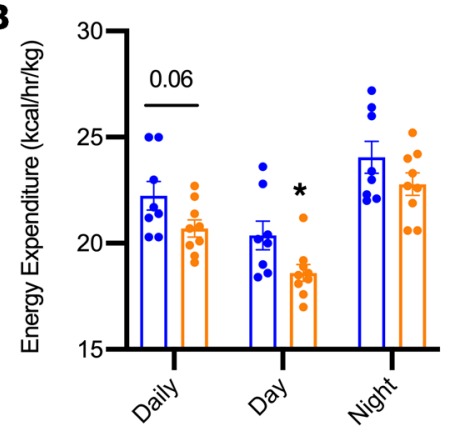

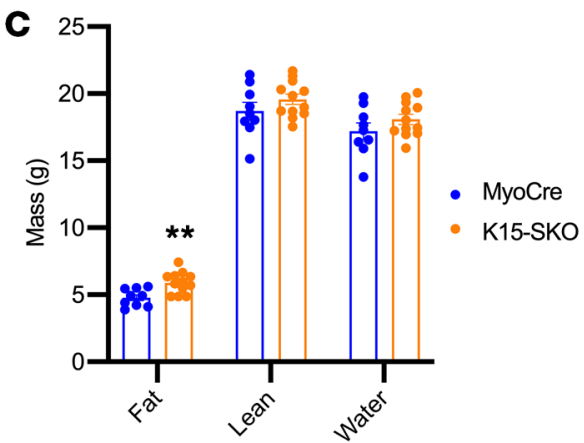
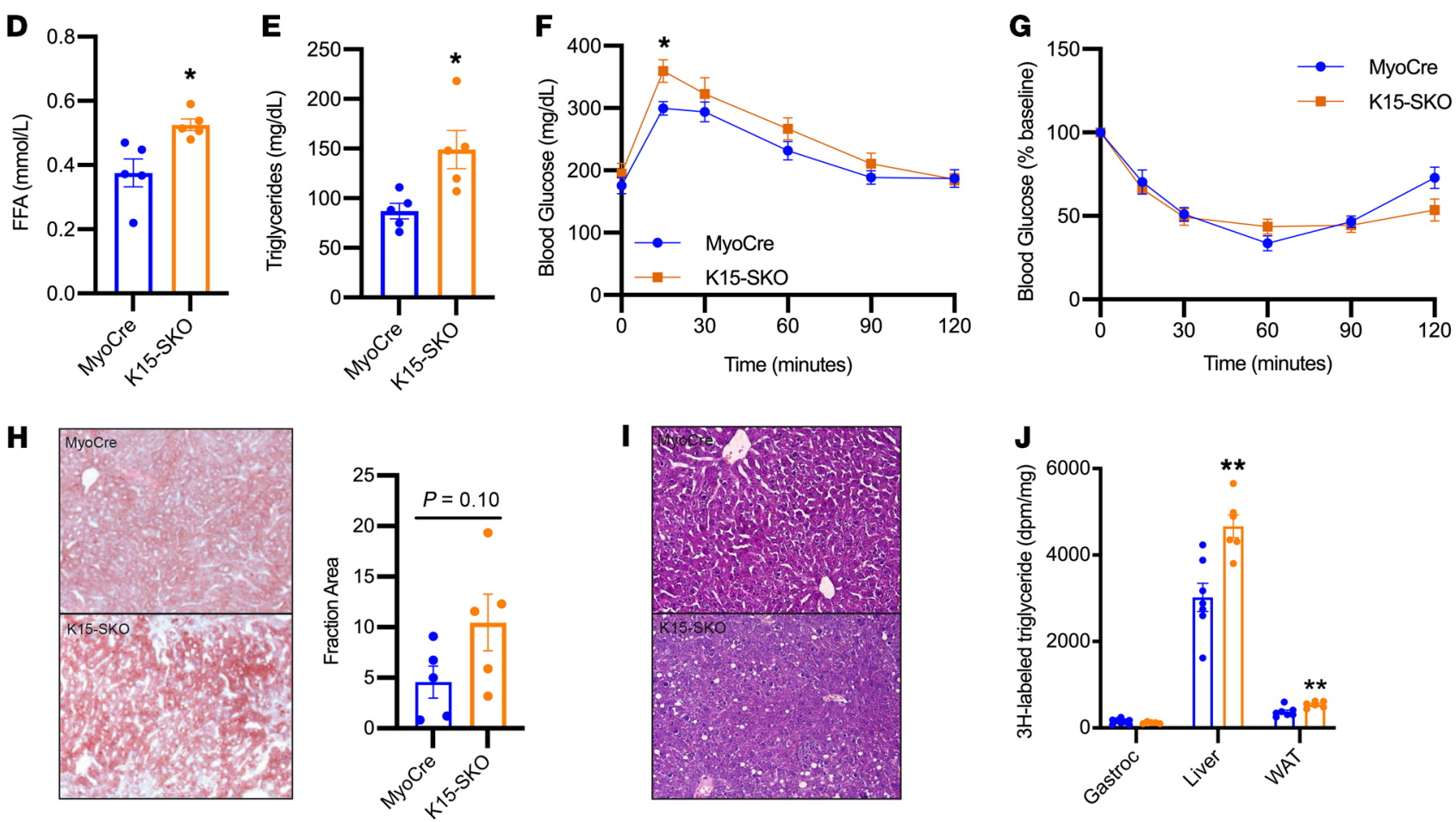

Figure 1. Skeletal muscle KLF15 regulates systemic metabolic homeostasis. (A) Body weight (g) of animals starting from 8 weeks of age $(n=13-15)$. (B) Daily, day, and night averages for energy expenditure $(n=8-9)$. (C) Body composition of fat, lean, and water mass as measured by quantitative magnetic resonance imaging ( $n=$ 9-12). (D) Plasma FFAs $(n=5)$. (E) Increased plasma triglycerides (TGs) in K15-SKO mice $(n=5)$. (F) IPGTT $(n=6-7)$. (G) IPITT ( $n=7)$. (H) Representative images of Oil Red $\mathrm{O}(\mathrm{ORO})$ liver staining at $\times 20$ and quantification of proportional area of positive staining. (I) Representative images of H\&E-stained sections of liver at $\times 20$, indicating steatosis in K15-SKO livers. (J) 3H-labeled palmitate tracing in gastrocnemius (gastroc), liver, and WAT ( $n=8-9$ ). Data represent mean \pm SEM. Comparisons between MyoCre and K15-SKO mice were performed using an unpaired, 2-tailed Student's $t$ test, ${ }^{*} P<0.05,{ }^{* *} P<0.01,{ }^{* * *} P<0.001$.

(MyoCre) line (Supplemental Figure 1C, and Supplemental Methods). Gene expression of skeletal muscle contractile apparatus components, muscle fiber cross-sectional area, fiber type distribution, mitochondrial quantity and morphology, and sarcomere morphology remain unaltered in K15-SKO mice (Supplemental Figure 1, D-G).

We first characterized skeletal muscle KLF15's impact on metabolism at baseline. K15-SKO mice consistently demonstrated increased weight gain on control diet (CD) starting at 8 weeks of age (Figure 1A). There were no observable differences in food intake, intestinal lipid absorption, or physical activity (Supplemental Figure 2, A-C), however K15-SKO mice showed decreased energy expenditure (Figure 1B), suggesting an alteration in basal metabolism. Quantitative magnetic resonance imaging revealed that the increase in body weight in K15-SKO mice is due to an increase in fat mass but not in lean or water mass (Figure 1C). Hematoxylin and eosin (H\&E) staining of white adipose tissue (WAT) showed adipocyte hypertrophy in K15-SKO mice (Supplemental Figure 2D). Concordantly, K15-SKO mice exhibited dyslipidemia with elevated plasma free fatty acids (FFAs) and triglycerides (Figure 1, D and E). Intraperitoneal glucose tolerance test (IPGTT) and intraperitoneal insulin tolerance test (IPITT) revealed developing glucose intolerance in K15-SKO mice, although accompanied by minimal changes in insulin sensitivity at baseline (Figure 1, F and G). Together, these studies illustrate that skeletal muscle KLF15 deficiency is metabolically deleterious, conferring a metabolic disease phenotype encompassing obesity, dyslipidemia, and glucose intolerance.

NAFLD is intimately linked with obesity and diabetes and currently represents the most common liver pathology in the Western world $(12,13)$. Interestingly, past studies have demon- 

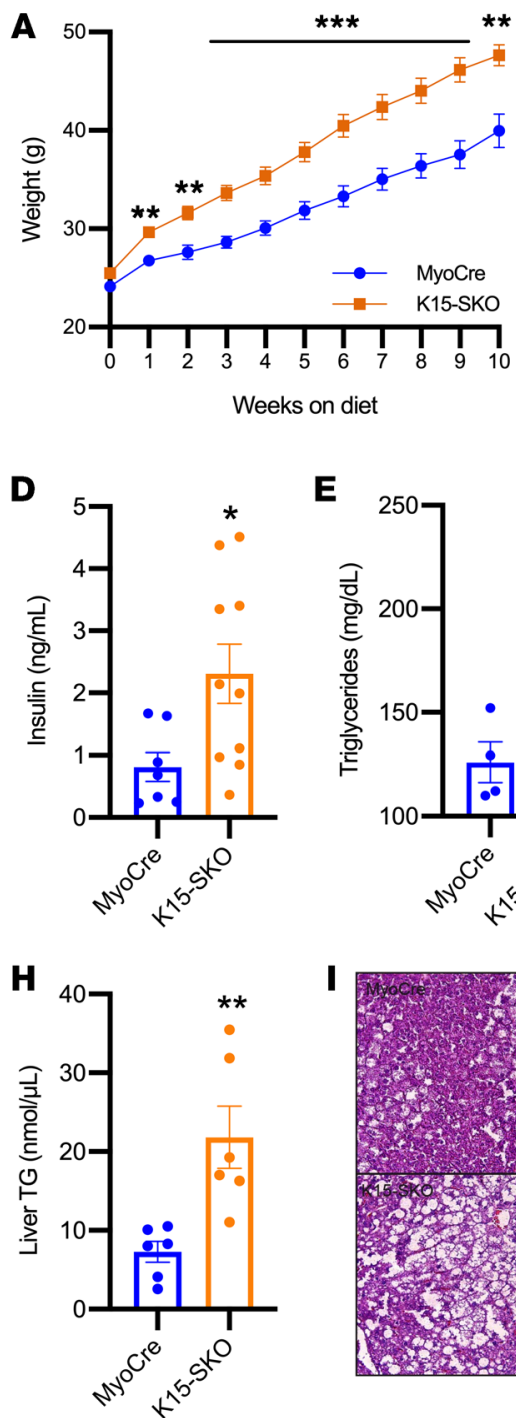
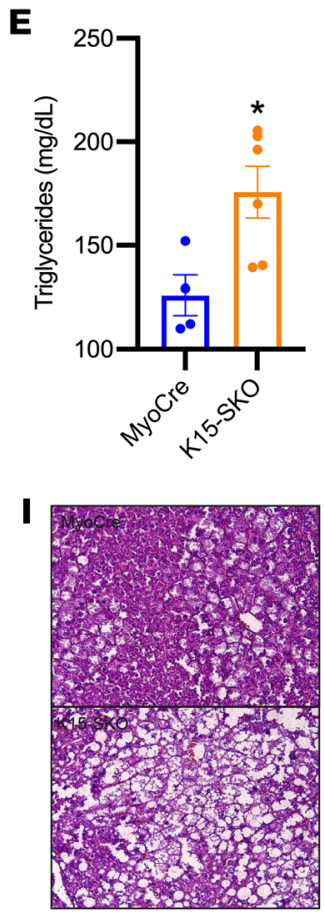
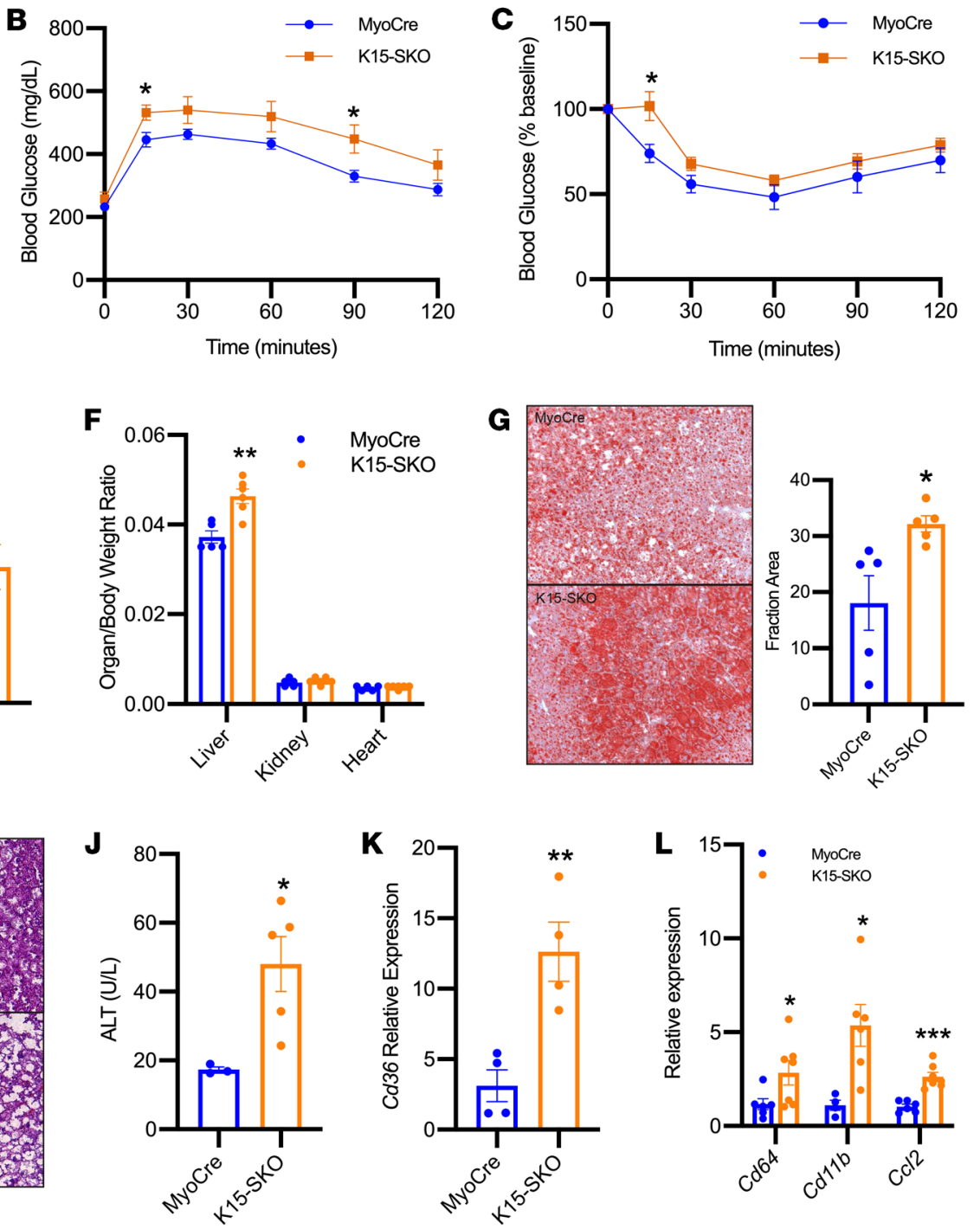

Figure 2. K15-SKO mice exhibit exacerbated metabolic disease phenotype with HFD. (A) Body weight (g) of animals on HFD starting from 8 weeks of age ( $n$ = 9-13). (B) IPGTT ( $n=7-8)$. (C) IPITT at 10 weeks on HFD ( $n=6-7)$. (D) Plasma insulin levels $(n=6-7)$. (E) Plasma triglyceride levels ( $n=4-6)$. (F) Organ to body weight ratio of liver, kidney, and heart $(n=5-6)$. (C) Representative images of liver ORO staining in MyoCre vs. K15-SKO mice on HFD and quantification of proportional area of positive staining. (H) Liver tissue TC $(n=6-8)$. (I) Representative images of H\&E-stained liver sections of MyoCre vs. K15-SKO mice. (J) Plasma alanine transaminase (ALT) concentration ( $n=3-5)$. (K) Liver Cd36 expression $(n=4)$. (L) Expression of various inflammatory genes in liver tissue $(n=6-7)$. All animals were on HFD for 10 weeks unless otherwise indicated. Data represent mean \pm SEM. Comparisons between MyoCre and K15-SKO mice were performed using an unpaired, 2-tailed Student's $t$ test, ${ }^{*} P<0.05,{ }^{* *} P<0.01,{ }^{* *} P<0.001$.

strated a relationship between skeletal muscle health and liver health. For example, myopathies and decreased skeletal muscle mass greatly increases the risk for liver steatosis; however, the mechanism underlying this phenomenon is largely unknown (24). We observed that K15-SKO mice showed a propensity toward developing NAFLD even at baseline with trending increased hepatic lipid deposition, micro- and macrovesicular steatosis, and increased Cd36 expression (Figure 1, H and I, and Supplemental Figure 2, E and F). Importantly, lipid tracing studies demonstrated a striking increase in $3 \mathrm{H}$-labeled palmitate deposition in the liver and WAT of K15-SKO mice at baseline (Figure 1j). These data suggest that skeletal muscle KLF15 may act as a novel extrahepatic regulator of classic metabolic disease and associated comorbidities.
While KLF15 variants associated with metabolic disease have been identified, the responsiveness of this important factor to pathological metabolic stimuli such as dietary excess is unknown $(10,11)$. Our studies demonstrate increased skeletal muscle Klf15 levels in both diet-induced and genetic metabolic disease models (Supplemental Figure 3, A and B). The observed elevation in skeletal muscle Klf15 may serve to activate compensatory metabolic programs to maintain homeostasis in the face of excess lipids or glucose, suggesting the protective role that skeletal muscle KLF15 may play against the pathogenesis of metabolic disorders.

To explore this role, we administered a high-fat diet (HFD) to MyoCre and K15-SKO mice for 10 weeks. K15-SKO mice became significantly obese through expansion of fat mass despite no changes in food intake (Figure 2A, and Supplemental 3, C-E). Addition- 
A
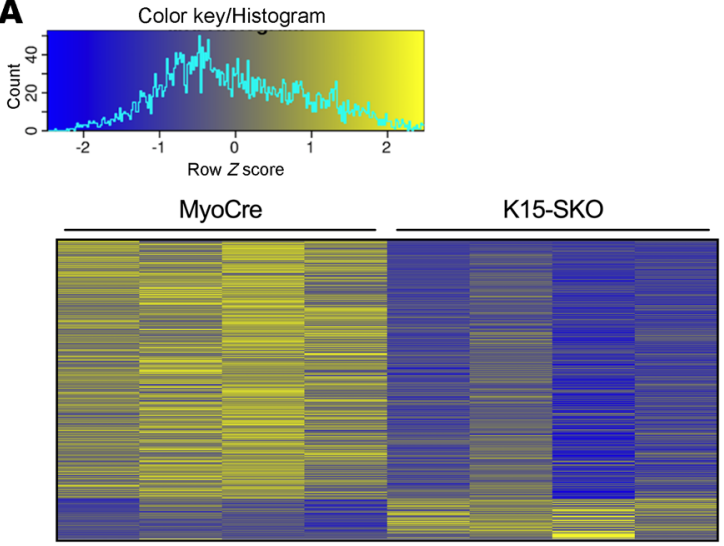

C

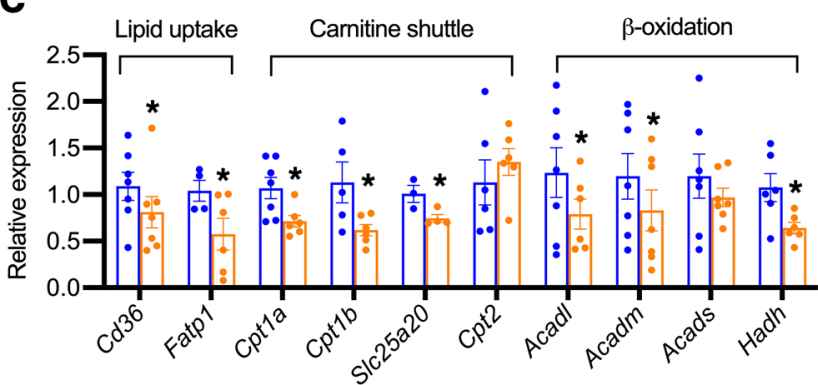

D

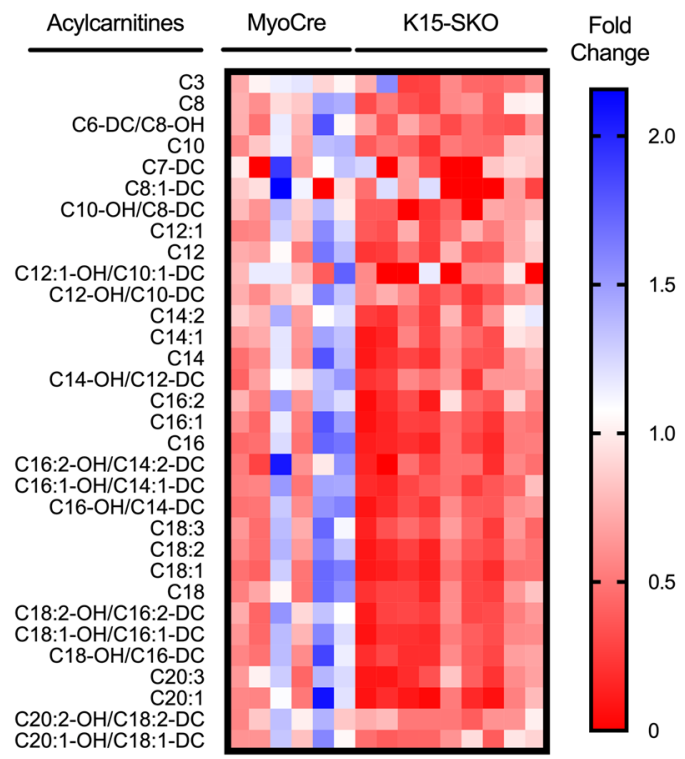

B
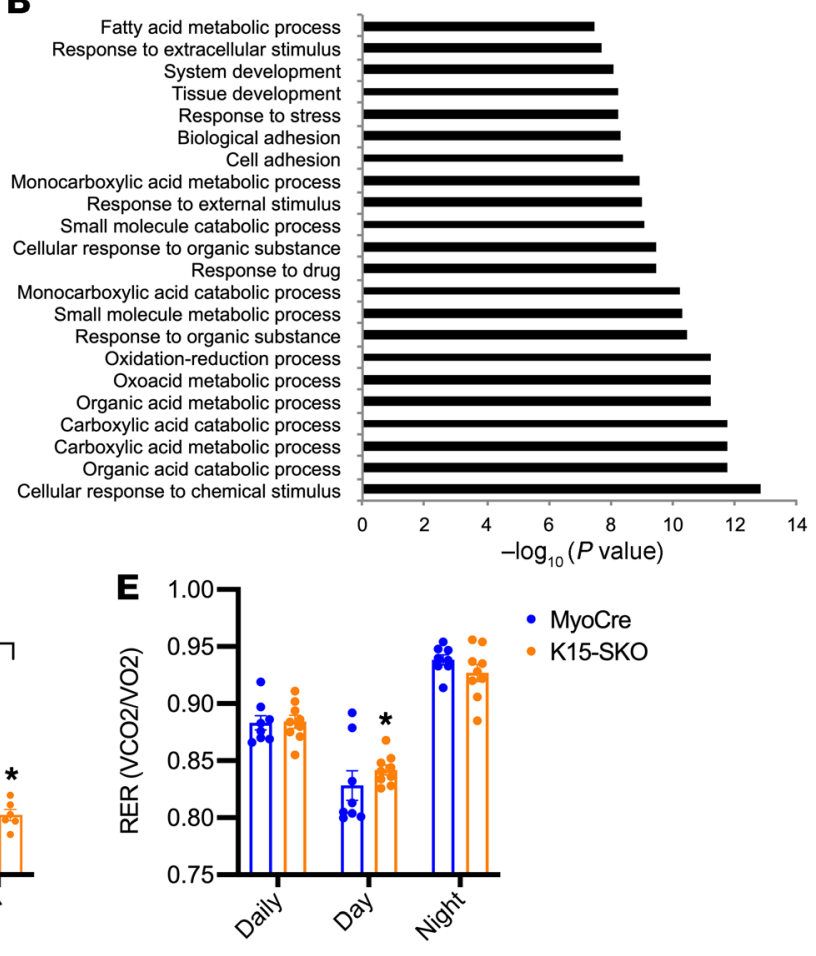

$\mathbf{F}$

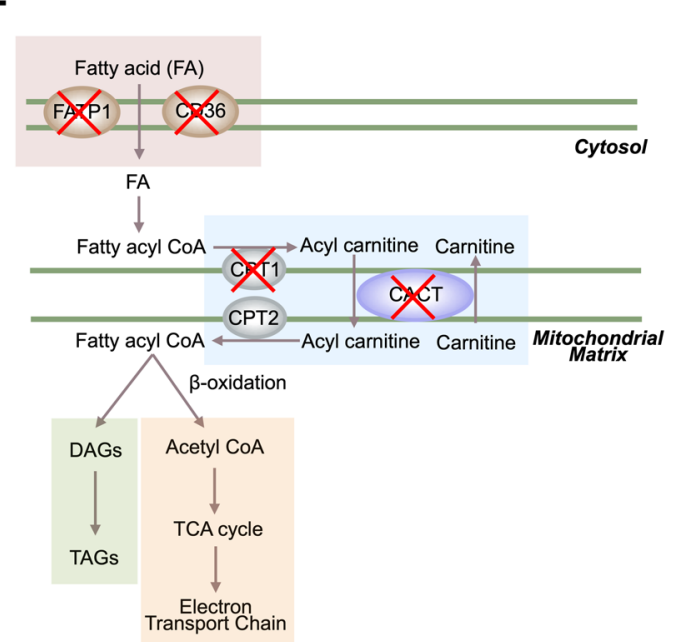

Figure 3. Skeletal muscle KLF15 is a major regulator of lipid flux pathways. (A) Heatmap clustering of 496 differentially enriched genes found in RNA sequencing of soleus muscle $(n=4)$. (B) Gene-set enrichment analysis using Gene Ontology Biological Processes showing the top 20 most significantly enriched pathways. (C) qPCR of select lipid flux pathway genes in soleus $(n=3-7)$. (D) Acylcarnitine analysis in soleus by tandem mass spectrometry. Data normalized to control group average for each acylcarnitine species and significantly different species $(P<0.05)$ are shown $(n=6-9)$. (E) Metabolic cage respiratory exchange quotient $($ RER) $(n=8-9)$. (F) Schematic representation of lipid flux pathway and defects seen in K15-SKO mice. Data represent mean \pm SEM. Comparisons between MyoCre and K15-SKO mice were performed using an unpaired, 2-tailed Student's $t$ test, ${ }^{*} P<0.05,{ }^{* *} P<0.01,{ }^{* *} P<0.001$.

ally, K15-SKO mice exhibited an enhanced diabetic phenotype with glucose intolerance (Figure 2B), insulin resistance (Figure 2C), higher circulating insulin levels (Figure 2D), and increased circulating triglycerides (Figure 2E). NAFLD in K15-SKO mice was markedly exacerbated as well. K15-SKO mice demonstrated hepatomegaly (Figure 2F), severe hepatic lipid deposition (Figure 2,
$\mathrm{G}$ and $\mathrm{H}$ ), hepatocellular ballooning and damage (Figure 2I), elevated alanine transaminase (ALT) levels (Figure 2J), and 12-fold higher expression of liver $C d 36$ (Figure 2K). Additionally, there is evidence of inflammation and NAFLD disease progression to nonalcoholic steatohepatitis (NASH, Figure 2L). These findings indicate that K15-SKO mice suffer from aberrant lipid handling by 
A
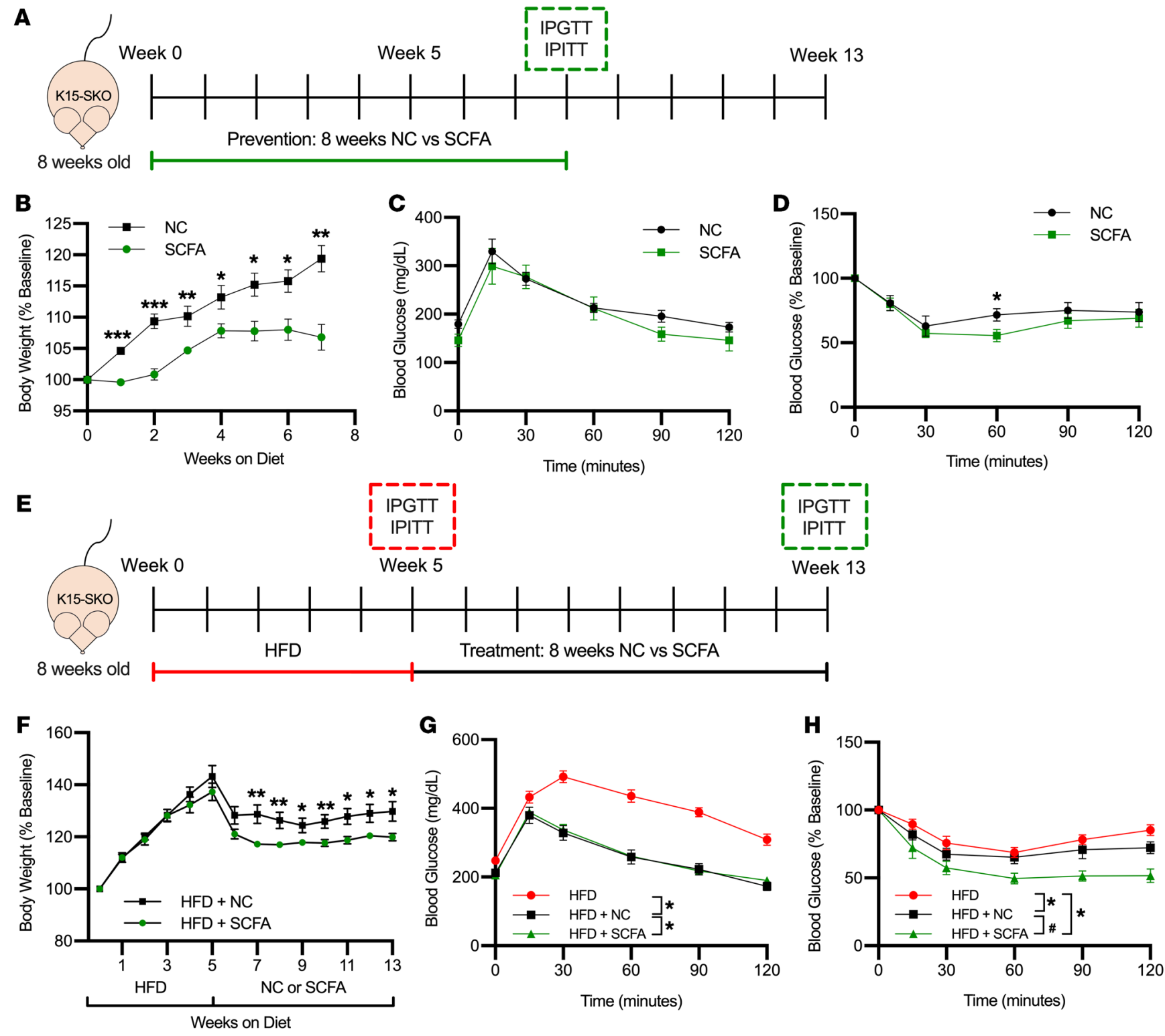

Figure 4. SCFA-rich diet ameliorates lipid handling defects in K15-SKO mice. (A) Schematic of prevention study pertaining to panels B-D depicting 8 weeks on either normal chow (NC) or SCFA-enriched diet followed by IPGTT/IPITT assessment. (B) Body weight of K15-SKO mice fed either NC or SCFA diet throughout 8 weeks of diet $(n=7-8)$. (C) IPGTT and (D) IPITT of K15-SKO mice fed either NC or SCFA $(n=5-7)$. (E) Schematic of therapy study pertaining to panels F-H depicting 5 weeks on HFD with IPGT/IPITT, followed by 8 weeks of either NC or SCFA diet and IPGTT/IPITT. (F) Body weight of K15-SKO mice fed 5 weeks of HFD followed by NC or SCFA diet for an additional 8 weeks $(n=9-10)$. (C) IPGTT and (H) IPITT after 5 weeks of HFD and after an additional 8 weeks of NC or SCFA diet $(n=6-11)$. Data represent mean \pm SEM. Comparisons between groups for panels B-D and F were performed using an unpaired, 2-tailed Student's $t$ test, ${ }^{*} P<0.05$, , ${ }^{* *} P<0.01$, , ${ }^{* * *} P<0.001$. Comparisons between groups in panels $\mathrm{F}-\mathrm{H}$ were performed using 1 -way ANOVA with Tukey's post hoc test, ${ }^{*} P<0.05$ compared with HFD, ${ }^{\#} P<0.05$ compared with HFD+NC.

skeletal muscle, leading to the accumulation of excess lipids and lipid intermediates in the circulation and consequent deposition into distant organs such as WAT and liver.

Given KLF15's importance in orchestrating metabolic networks in diverse tissues, we sought to determine if perturbation of metabolic transcriptional signatures could explain the deficits in lipid handling noted in K15-SKO mice. RNA sequencing of soleus revealed 496 differentially expressed genes, the majority of which are downregulated in K15-SKO mice and many of which are part of lipid metabolism pathways (Figure 3, A and B). We confirmed these findings through RNA sequencing of quadriceps muscle as well (Supplemental Figure 4). We assessed the expression of a number of genes involved in the tightly coordinated steps of the skeletal muscle lipid flux pathway. Genes encoding for transporters of FFA into the cell, Fatp1 and Cd36, are downregulated in K15SKO mice (Figure 3C). While certain lipid species can easily pass through the mitochondrial membrane (e.g., SCFAs), long-chain fatty acids (LCFAs) need to undergo conversions into acyl-CoAs and then acylcarnitines followed by transport by the mitochondrial carnitine shuttle. We observed downregulation of several genes that encode for components of the carnitine shuttle, including Cpt1a, Cpt1b, and Slc25a2O (Figure 3C). Additionally, we found 
decreased expression of genes involved in the oxidation of longand medium-chain fatty acids (e.g., Acadl and Acadm, respectively), likely as a response to the decreased levels of LCFAs transported into the mitochondria (Figure 3, C and F).

Given the downregulation of genes involved in cellular uptake, movement into the mitochondria, and conversion of LCFAs into acylcarnitines, we expected to see a decrease in the concentration of muscle acylcarnitines. Indeed, acylcarnitine analysis of soleus by tandem mass spectrometry revealed a significant paucity of longchain acylcarnitines in K15-SKO mice (Figure 3D). Paired with the skeletal muscle lipid tracing data (Figure 1J), it appears that although lipid transport into the cell is downregulated, the major defect in the lipid flux pathway lays within the mitochondrial transport machinery. Concordant with these observed FA metabolism defects, functional readouts using metabolic cages revealed a higher respiratory exchange ratio (RER, Figure 3E) and lower $\mathrm{VO}_{2}$ (Supplemental Figure 4, B and C) in K15-SKO mice, signifying increased reliance on carbohydrates rather than lipids for energy. Taken together, these studies underline a critical role for skeletal muscle transcriptional regulators in maintaining systemic lipid homeostasis and highlight the importance of skeletal muscle as a lipid metabolism organ on par with both the liver and WAT.

In humans, several inborn errors of metabolism have been identified that stem from defects in the components of the carnitine shuttle. Individuals who harbor mutations in Cpt1, Slc25a2O, or Cpt2 exhibit a phenotype that manifests as an inability to process LCFA, severe hypoglycemia upon fasting, and hypoketosis along with various organ pathologies (e.g., seizures, arrhythmias, etc.) (14). Management of these disorders includes consumption of diets high in carbohydrates and avoidance of diets high in LCFAs. Recently, it has been reported that a diet rich in SCFAs can bypass defects in the carnitine shuttle and ultimately improve both organ function and overall metabolic health $(15,16)$.

Given the specific lipid flux pathway defects we see in K15SKO mice, we sought to explore SCFA-enriched diets as a potential preventative strategy against the development of metabolic disease and as a therapeutic avenue to ameliorate the observed metabolic disease phenotype. To assess the efficacy of SCFA diets as a prevention, K15-SKO mice were fed either normal chow (NC) or SCFA diet for 8 weeks starting at 8 weeks of age (Figure $4 \mathrm{~A}$ ). K15-SKO mice fed a SCFA diet had significantly decreased weight gain compared with those fed NC (Figure 4B). While there were minimal changes in glucose intolerance at this time point, K15SKO mice fed SCFA diet did have improvements in insulin sensitivity, which was surprising and promising given the modest glucose handling deficits at baseline in K15-SKO mice (Figure 4, C and D). Gene expression studies in soleus only showed a modest trend toward increased expression of a few lipid oxidation genes, suggesting that the diet's observed effect is likely due to a mechanism separate from transcriptional control (Supplemental Figure $5 \mathrm{~A}$ ). These results indicate that SCFA diets can be effective in delaying the development of obesity and associated insulin insensitivity resultant of impaired lipid handling.

We next sought to determine if SCFA diets could impart metabolic benefits in the setting of existing metabolic disease. To this end, we gave K15-SKO mice HFD for 5 weeks to stimulate diet-induced obesity, followed by 8 weeks of either NC (denoted
HFD+NC) or SCFA (HFD+SCFA) diet to determine the efficacy of SCFA in treating the obesity phenotype (Figure 4E). Animals following the HFD+SCFA regimen experienced a more dramatic decrease in body weight (Figure $4 \mathrm{~F}$ and Supplemental 5B). Mice were assessed for glucose tolerance and insulin sensitivity at the end of 5 weeks of HFD and again after the additional 8 weeks of NC or SCFA. K15-SKO mice fed HFD+SCFA showed significantly improved glucose tolerance compared with before starting SCFA (Figure 4G), and demonstrated improvements in insulin sensitivity compared with both pre-SCFA diet and to mice fed HFD+NC (Figure $4 \mathrm{H}$ ). There is also a trend toward improved hepatic health, with lower plasma ALT and decreased hepatic lipid deposition (Supplemental Figure 5, C and D). Similar to the prevention study, no significant differences were found in the expression of various lipid and glucose metabolism genes between mice fed HFD+NC and those fed HFD+SCFA (Supplemental Figure 5E). Taking both the prevention and treatment studies together, these data strongly suggest that SCFA-rich diets are a promising candidate for combating metabolic diseases that arise due to impaired skeletal muscle lipid handling.

Collectively, these observations underscore a vital role for skeletal muscle in the control of systemic lipid and metabolic homeostasis. We note that, to date, the bulk of published work has focused on skeletal muscle's role in metabolic syndrome as a driver of insulin resistance, which then has negative downstream effects on liver and WAT (17). Here, we show that a primary defect in skeletal muscle (i.e., loss of KLF15) can contribute to increased lipid deposition in distant organs even in the basal (chow-fed) state characterized by mild glucose intolerance and insulin insensitivity, thus offering additional insight as to how skeletal muscle can contribute to the development of metabolic disease. Furthermore, these findings have implications for metabolic sequelae seen in myopathic disease states. For example, patients with Duchenne muscular dystrophy and spinal muscular atrophy have a higher propensity to develop NAFLD. Interestingly, both conditions are associated with reduced skeletal muscle KLF15 expression patterns (18-20). As such, this study also underscores the vital role KLF15 plays in orchestrating nutrient flux across diverse organs and emphasizes its importance as a nodal regulator of systemic metabolic homeostasis.

\section{Methods}

Complete details on experimental methods are provided in the Supplemental Materials.

Statistics. Statistical analyses were performed using GraphPad Prism software. Data are represented as mean \pm SEM ( $n$ as indicated in figure legends). Statistical evaluation of groups was performed by either 2-tailed Student's $t$ test or 1-way ANOVA with Tukey's post hoc test. Statistical significance was defined as $P$ less than 0.05 .

RNA sequencing. The RNA sequencing data discussed in this publication have been deposited in NCBI's Gene Expression Omnibus. The soleus and quadriceps RNA sequencing data sets can be accessed through numbers GSE160848 and GSE160877, respectively. See Supplemental Methods for more details.

Study approval. All animal studies were approved by the Case Western Reserve University's Institutional Animal Care and Use Committee. 


\section{Author contributions}

LF, DRS, DAP, and MKJ designed the studies. LF, DRS, DAP, RZ, YL, OI, KSK, and CEB performed the experiments. LF, VV, and ERC analyzed the data. DRS, DAP, YL, CBN, and MJK provided critical research guidance. $L F$ prepared the figures and drafted the manuscript. DRS and MKJ edited the manuscript. MKJ approved the final version of the manuscript.

\section{Acknowledgments}

This work was supported by NIH grants R35HL135789 and R01HL086548 (to MKJ), R01DK111478/RES511821 (to MKJ and CBN), T32GM007250 (to LF and DRS), T32HL134622 (to LF), and F30HL139014 (to DRS). This work was also supported by the Amer- ican Heart Association (AHA) Established Investigator Award and the AHA-Allen Frontiers Award (to MKJ) and the Leducq Foundation Transatlantic Network of Excellence (to MKJ). Part of this study was performed at the National MMPC at University of Massachusetts Medical School (5U2C-DK093000), the University Cincinnati MMPC Center (U2C DK059630), Case Western Reserve University MMPC, and Case Western Reserve University Cryo-Electron Microscopy Core.

Address correspondence to: Mukseh K. Jain, Case Western Reserve University, School of Medicine, Cardiovascular Research Institute, Wolstein Research Building, 2103 Cornell Road, WRB 4-522, Cleveland, Ohio 44106 USA. Phone: 216.368.3607; Email: mxj84@case.edu.
1. Argilés JM, et al. Skeletal muscle regulates metabolism via interorgan crosstalk: roles in health and disease. J Am Med Dir Assoc. 2016;17(9):789-796.

2. Moon JS, et al. The role of skeletal muscle in development of nonalcoholic fatty liver disease. Diabetes Metab J. 2013;37(4):278-285.

3. Bhanji RA, et al. Sarcopenia in hiding: The risk and consequence of underestimating muscle dysfunction in nonalcoholic steatohepatitis. Hepatology. 2017;66(6):2055-2065.

4. Kim G, et al. Relationship between relative skeletal muscle mass and nonalcoholic fatty liver disease: a 7-year longitudinal study. Hepatology. 2018;68(5):1755-1768.

5 . Shieh K, et al. Frequency and predictors of nonalcoholic fatty liver disease in myotonic dystrophy. Muscle Nerve. 2010;41(2):197-201.

6. Gray S, et al. The Krüppel-like factor KLF15 regulates the insulin-sensitive glucose transporter GLUT4. J Biol Chem. 2002;277(37):34322-34328.

7. Hsieh PN, et al. The Krüppel-like factors and control of energy homeostasis. Endocr Rev. 2019;40(1):137-152.
8. Jeyaraj D, et al. Klf15 orchestrates circadian nitrogen homeostasis. Cell Metab. 2012;15(3):311-323.

9. Fan L, et al. Krüppel-like factor 15: Regulator of BCAA metabolism and circadian protein rhythmicity. Pharmacol Res. 2018;130:123-126.

10. Patel SK, et al. Genetic variation in kruppel like factor 15 is associated with left ventricular hypertrophy in patients with type 2 diabetes: discovery and replication cohorts. EBioMedicine. 2017;18:171-178.

11. Pettersson $\mathrm{M}$, et al. Copy number variants are enriched in individuals with early-onset obesity and highlight novel pathogenic pathways. J Clin Endocrinol Metab. 2017;102(8):3029-3039.

12. Browning JD, et al. Prevalence of hepatic steatosis in an urban population in the United States: impact of ethnicity. Hepatology . 2004;40(6):1387-1395.

13. Loomba R, Sanyal AJ. The global NAFLD epidemic. Nat Rev Gastroenterol Hepatol. 2013;10(11):686-690.

14. El-Gharbawy A, Vockley J. Inborn errors of metabolism with myopathy: defects of fatty acid oxidation and the carnitine shuttle system. Pedi- atr Clin North Am. 2018;65(2):317-335.

15. York B, et al. Ablation of steroid receptor coactivator-3 resembles the human CACT metabolic myopathy. Cell Metab. 2012;15(5):752-763.

16. Sugi $\mathrm{K}$, et al. Kruppel-like factor 15 is required for the cardiac adaptive response to fasting. PLOS One. 2018;13(2):e0192376.

17. Shulman GI. Ectopic fat in insulin resistance, dyslipidemia, and cardiometabolic disease. $N$ EnglJ Med. 2014;371(23):2237-2238.

18. Morrison-Nozik A, et al. Glucocorticoids enhance muscle endurance and ameliorate Duchenne muscular dystrophy through a defined metabolic program. Proc Natl Acad Sci U S A. 2015;112(49):E6780-E6789.

19. Deguise M, et al. Abnormal fatty acid metabolism is a core component of spinal muscular atrophy. Ann Clin Transl Neurol. 2019;6(8):1519-1532.

20. Walter LM, et al. Interventions targeting glucocorticoid-Krüppel-like factor 15-branched-chain amino acid signaling improve disease phenotypes in spinal muscular atrophy mice. EBioMedicine. 2018;31:226-242. 